Entropy 2005, 7[3], 188-189

Entropy

ISSN 1099-4300

www.mdpi.org/entropy/

Editorial

\title{
The FIS2005 Conference in Paris
}

\section{Michel Petitjean}

ITODYS (CNRS, Université Paris 7, UMR 7086), 1 rue Guy de la Brosse, 75005 Paris, France E-mail: petitjean@itodys.jussieu.fr

Received: 15 July 2005 / Accepted: 19 July 2005 / Published: 19 July 2005

The FIS2005 - Third Conference on the Foundations of Information Science - has been held in Paris, 4-7 July 2005. The web site of the Conference is on the MDPI server:

$$
\text { http://www.mdpi.org/fis2005 }
$$

Following the spirit of the FIS (Foundations of Information Science), the major originality of the Conference was its pluridisciplinarity, allowing scientists, philosophers and artists to discuss together about the multiple facets of information. There were 115 contributors, including 76 attendees and 91 authors or coauthors, originating from 28 countries over the 5 continents, including a delegation from the UNESCO.

The Conference was sponsored by the following institutions: CNRS (France), DGA (France), ENSTA (France), ICT\&S (Austria), Kalamazoo College (USA), and University Paris 7 (France). Informations about the sponsors are available at http://www.mdpi.org/fis2005/sponsors.html

The proceedings (http://www.mdpi.org/fis2005/proceedings.html) are publihsed electronically in open access by MDPI. We count 69 publications (36 abstracts plus 33 papers), associated to 9 invited lectures, 34 contributed lectures, and 27 posters communications. All papers have been anonymously peer reviewed, and were associated indifferently to oral communications or to posters.

The Conference programme was divided into thematic sessions, given below in chronological order:

- The physics session, chaired by Francis Muguet, then by Michel Petitjean

- The chemistry session, chaired by Michel Petitjean

- The "bio" session, chaired by Gordana Dodig-Crnkovic, then by Koichiro Matsuno

- The maths/computer sciences session, chaired by John Collier, then by Ted Goranson 
- The round table on Interdisciplinary Teaching and Information Science, chaired by Péter Érdi. The participants were: Soren Brier, Wolfgang Hofkirchner, Bernd-Olaf Küppers, Kevin Kirby, Marcin Jan Schroeder, and Hans Christian von Baeyer.

- The posters session

- The general session, chaired by Dail Doucette, Soren Brier, and then by Wolfgang Hofkirchner.

Thermodynamical entropy and informational entropy were of course evoked in several sessions. Despite it is a small part of the field of information, they gave rise to discussions during the Conference, as well as on the FIS forum [1].

The names given to the sessions are highly reductionist, and give a poor idea of the richness of the contributions and of the discussions who occurred during each session. I have been very impressed by the high motivation of the participants, and by the fruitful dialog between specialists of information originating from so different fields.

The closure was done by Koichiro Matsuno, Pedro Marijuán, Michel Petitjean and then by Francis Muguet. The Friday morning after the Conference, the attendees were invited to a working session chaired by Dail Doucette, who initiated the project of creating the "Science of Information Institute".

My personal conclusion is that the FIS and MDPI should continue their fruitful collaboration initiated in 2002 [2,3], to promote the open access to scientific information.

Acknowledgments: I thank very much Pedro Marijuán for having incited me to organize the FIS2005 Conference, and for having managed the review of this editorial. Many thanks to my friends of the FIS2005 Committee for their invaluable help, and to all the participants of the Conference: the success of FIS2005 is due to them.

\section{References and notes}

[1] FIS Session on "Entropy and information", 2004

http://fis.icts.sbg.ac.at/fisspecials/index.html

This session has given rise to the publication of a special issue in the journal

Symmetry: Culture and Science, 2005, 16[3], 112 pages; M. Petitjean, Guest Editor.

http://www.geocities.com/symmetrion/isa/journal.htm

[2] Pedro C. Marijuán and Shu-Kun Lin

Editorial: Papers from the Foundations of Information Science 2002 (FIS 2002)

Entropy, 2003, 5[1], 1-2 (http://www.mdpi.org/entropy/htm/e5010001.htm)

[3] Pedro C. Marijuán

Foundations of Information Science. Selected papers from FIS 2002

Entropy, 2003, 5[2], 214-219 (http://www.mdpi.org/entropy/htm/e5020214.htm)

(c)2005 by MDPI (http://www.mdpi.org). Reproduction for noncommercial purposes permitted. 